Check for updates

Cite this: Phys. Chem. Chem. Phys., 2020, 22, 26552

Received 15th September 2020, Accepted 29th October 2020

DOI: $10.1039 / \mathrm{d} 0 \mathrm{cp} 04886 \mathrm{k}$

rsc.li/pccp

\section{Interactions of selected organic molecules with a blue phosphorene monolayer: self-assembly, solvent effect, enhanced binding and fixation through coadsorbed gold clusters}

\author{
T. Gorkan, ${ }^{a}$ Y. Kadioglu, (D) ${ }^{a}$ E. Aktürk (D) *ab and S. Ciraci (D) *c
}

In this paper we investigate the interaction between a pristine blue phosphorene monolayer and selected organic molecules like amino acids and nucleic acid bases. These molecules are bound to the substrate by a weak van der Waals interaction leading to their physisorption. When isolated, they tend to orient themselves parallel to the surface and are located in flat minima with very low libration frequencies; thus the electronic structures of the substrate and physisorbed molecules are not affected except for relative shifts. Even though the regular self-assembly of these molecules on the pristine blue phosphorene cannot be realized under this weak interaction, only their irregular coating of the substrate can occur due to increased intermolecular coupling. In a solvent like water, the weak binding energy is further decreased. Gold adatoms and gold clusters can form strong chemical bonds with pristine blue phosphorene and modify its electronic and magnetic state depending on the coverage. While full coverage of a blue phosphorene monolayer by gold adatoms leads to instabilities followed by clustering, relatively lower coverage can attribute very interesting magnetic and electronic states, like a spin gapless semiconductor. When bound to the gold clusters already adsorbed on the blue phosphorene monolayer, amino acid and nucleic acid base molecules form relatively strong chemical bonds and hence can be fixed to the surface; they are reoriented to gain self-assembly character and the whole system acquires new functionalities.

\section{Introduction}

Interfaces between biological molecules and two-dimensional (2D) inorganic nanostructures have been a subject of active research because of their applications in medical diagnostics, cancer therapy, cytotoxicity, vaccines etc. ${ }^{1-12}$ The integration of biomolecules such as DNA, proteins, peptides, viruses etc. with these materials has opened a new field in nanoscience and biomedicine. ${ }^{13-15}$ Through these interfaces, 2D materials and monolayers of selected materials can be functionalized for bio-integrated electronics, bio-medical applications like biosensors and smart drugs. ${ }^{16-19}$ While DNA and polynucleotides are gaining vital importance in genomics, diagnosis and treatments of diseases, particularly in diverse cancers, organic molecule biosensors which can provide a biological recognition mechanism, have played a critical role in the detection of

\footnotetext{
${ }^{a}$ Department of Physics, Adnan Menderes University, 09100 Aydın, Turkey. E-mail: ethem.akturk@adu.edu.tr; Fax: +902562135379; Tel: +902562130835

${ }^{b}$ Nanotechnology Application and Research Center, Adnan Menderes University, 09100 Aydin, Turkey

${ }^{c}$ Department of Physics, Bilkent University, 06800 Ankara, Turkey.

E-mail: ciraci@fen.bilkent.edu.tr
}

microorganisms or diagnostics. ${ }^{20,21}$ In this respect, 2D materials, like graphene, silicene, monolayer $\mathrm{MoS}_{2}, \mathrm{WS}_{2}, \mathrm{~h}-\mathrm{BN}$, graphene nanoribbons, and silicon nanowires, ${ }^{22-27}$ are used for DNA base and larger amino acid detection. For cases requiring stronger binding and hence fixation of organic molecules on the surface, patterned defects and adspecies can be created to ensure active sites to bind these molecules to the surface. ${ }^{12,28-31}$ Gold nanoparticles $\left(\mathrm{Au}_{n}\right)$ are shown to be effective in the detection of cancer cells $^{32}$ and in DNA immobilization. ${ }^{33}$

Organic molecules, which cover the surfaces of monolayers or multilayers of $2 \mathrm{D}$ materials can modify also the electronic and optical properties of surfaces. ${ }^{31,34-36}$ Owing to their chemical inertness, mechanical stability and high surfacevolume ratio, graphene and graphene based materials being the preliminary structure of $2 \mathrm{D}$ materials have been widely studied in biomedical applications. ${ }^{12,37-40}$ Additionally, graphene and similar materials have been utilized as bare and flat templates for organic molecules to synthesize larger molecules. ${ }^{41,42}$ While amino acids are analyzed by various experimental and theoretical studies, ${ }^{4-46}$ glycine, for example as a typical amino acid (AA) molecule, has been investigated actively because it acts as an inhibitory neurotransmitter in the central nervous system in 
the brain. ${ }^{47}$ Very recently, metal-insulator transition and heterostructure formation by glycine self-assembled on defect patterned graphene have been demonstrated. ${ }^{12}$ Beyond biological applications, it has also been predicted that on the coverage of graphene by self-assembled glycine the solar absorbance is enhanced significantly for photovoltaic applications. ${ }^{36}$ Graphene oxide (GO) and its derivatives exhibit critical properties that are used to carry DNA, proteins, small drug molecules etc. $^{37}$ The fact that the anticancer molecule doxorubicin (DXR) can form a strong bond with the GO surface and thus it can be used for drug carrier and biosensor applications has made GO suitable for tumor targeting. ${ }^{48,49}$ Similarly, blood proteins and insulin can be adsorbed on GO surfaces. ${ }^{50,51}$ On the other hand, latent cytotoxicity concerns ${ }^{51}$ and unusually high levels of toxicity and random scission of $\mathrm{DNA}^{37}$ on graphene and graphene based materials have directed interests towards different nanomaterials.

As an alternative to graphene, semiconducting black phosphorene $(\alpha-\mathrm{P})$ is used as a template for the self-assembly of DNA/RNA constituents. Interactions of amino acids and nucleic acid bases (nucleobases) with phosphorene were investigated for biomedical applications. ${ }^{9,10,31}$ The interaction of some amino acids with the $\alpha-\mathrm{P}$ surface has been investigated ${ }^{9,10}$ and compared with the surface of graphene in a honeycomb structure. Recent studies show that biomolecules interact with phosphorene via physisorption; nonetheless their binding can be enhanced through their adsorption to specific foreign adatoms on the surface of phosphorene. As compared to graphene, organic molecules on $\alpha$-P undergo relatively less deterioration. Hence, $\alpha-\mathrm{P}$ offers more biofriendly behaviors. Another 2D monolayer in the phosphorene-family, blue phosphorene $(\beta-\mathrm{P})$ with a buckled honeycomb structure was also synthesized. ${ }^{52}$ It is a stable, indirect band gap semiconductor, ${ }^{53}$ and exhibits many interesting physical properties suitable for diverse electronic, spintronic and biological applications.

In this paper, we investigate the interaction of amino acids, AA (Glutamine, Histidine, Tyrosine), and nucleic acid bases, NB (Adenine, Guanine, Thymine), with pristine/bare and gold cluster covered $\beta-\mathrm{P}$ monolayers using $a b$ initio density functional theory. Our objective is to seek answers to the following questions and reveal the underlying physics: (i) is the interaction between $\beta$-P and any of these molecules strong enough to fix it on the surface so that regular coverage/decoration of it can attain self-assembly behaviour and hence lead to new functionalities? (ii) How can the binding of the adsorbed molecule be affected by a usual solvent? (iii) Can the binding of any individual molecule be enhanced by the foreign adatoms or clusters adsorbed on the $\beta$-P surface? (iv) How can the physical properties of the bare surface of $\beta$-P covered by specific foreign atoms or their clusters be modified and how can the latter surface eventually be modified by the adsorption of these AA or NB molecules? To this end, we determined the equilibrium binding configurations and binding energies of these six, isolated molecules on bare $\beta$-P using a supercell geometry. To investigate the rotational dynamics of these physisorbed, isolated organic molecules and to reveal also their self-assembly behavior, we selected guanine, which has the strongest binding energy among AA and NB molecules studied in this paper, as a prototype example. By applying small angle rotations around the global minimum of physisorbed guanine we unveiled average parameters pertaining to its rotational dynamics. We than analyzed whether the optimized equilibrium configuration of guanine can be reproduced after small rotations/displacements and after a simulated annealing process. The effect of the solvent, considered as water, on the binding energy is also investigated. The modification of the electronic and magnetic properties of bare $\beta$-P by the decoration of single gold adatoms in different coverages, as well as by the dilute coverage of gold clusters, is revealed, and finally, the interaction of AA and NB molecules with these adsorbed clusters is investigated.

Several important results of our work will be detailed in the forthcoming sections and summarized by way of conclusions at the end. Here we point out only the critical and novel findings, which are absent in similar previous studies, in particular in those which investigated other phosphorene allotropes: simulated annealing studies demonstrate that the weak van der Waals interaction between the $\beta$-P monolayer and individual organic molecules studied here cannot induce restoring torques strong enough to sustain the equilibrium configuration against the thermal excitations. Accordingly, the libration frequencies become rather low. Under these circumstances, the regular self-assembly character of these organic molecules physisorbed to the $\beta$-P monolayer cannot be realized. Only the irregular coating of the substrate can occur due to increased molecule-molecule coupling. In a solvent like water, the interaction between these organic molecules and the underlying substrate is weakened. We showed how one can determine the binding energy of these molecules physisorbed to a substrate in a solvent. Further to the fixation of the organic molecules to the phosphorene monolayer through coadsorbed gold adatoms or clusters, we unveiled new and technologically important functionalities of the phosphorene monolayer attained through the coverage of gold adatoms. While full coverage of a $\beta$-P monolayer by gold adatoms leads to instabilities followed by clustering, relatively lower coverages can attribute very interesting magnetic and electronic properties, which were not known before.

\section{Methods}

Our theoretical study is carried out using the $a b$ initio density functional theory (DFT), which was demonstrated by previous studies to be powerful and, at the same time, accurate to unveil the details of interaction between an organic molecule and a 2D flat surface or monolayers of inorganic materials. The present DFT calculations were carried out by using the VASP package $\mathrm{e}^{54,55}$ within the generalized gradient approximation (GGA). Exchange-correlation potential ${ }^{56}$ is represented by PerdewBurke-Ernzerhof (PBE) functionals with the PAW pseudopotentials. ${ }^{57,58}$ The van der Waals (vdW) interaction was included by D2-Grimme correction (DFT-D2 $)^{59}$ in all calculations. Planewave basis sets with the kinetic energy cut-off value of $600 \mathrm{eV}$ were 
employed. The $k$-point mesh of $3 \times 3 \times 1$ was used for the Brillouin zone (BZ) integration. The energy convergence criterion of the electronic self-consistency was taken as $10^{-5} \mathrm{eV}$ between two successive iterations. A Gaussian type Fermi-level smearing method was used with a smearing width of $0.01 \mathrm{eV}$. In order to mimic the interaction between an isolated, individual molecule and the $\beta$-P monolayer, calculations were carried out using $(5 \times 5)$ supercells of $\beta$-P, where the spacing between $\beta$-P planes is taken to be $20 \AA$ to avoid interactions between images.

The binding energy, which measures the strength of the interaction between molecules (M) and the $\beta$-P monolayer, was calculated from the following expression,

$$
E_{\mathrm{b}}=E_{\mathrm{T}}[\beta-\mathrm{P}]+E_{\mathrm{T}}[\mathrm{M}]-E_{\mathrm{T}}[\beta-\mathrm{P}+\mathrm{M}]
$$

in terms of the total energy of the bare $\beta$-P per supercell $E_{\mathrm{T}}[\beta-\mathrm{P}]$, of free molecules $E_{\mathrm{T}}[\mathrm{M}]$ and of the $\beta$-P supercell having one molecule adsorbed $E_{\mathrm{T}}[\beta-\mathrm{P}+\mathrm{M}]$, respectively. Positive values of $E_{\mathrm{b}}$ indicate the binding. The binding (or cohesive) energy of free gold clusters, $\mathrm{Au}_{n}$, with $n=1-5$, per $\mathrm{Au}$ atom is calculated in terms of the total energy of a single $\mathrm{Au}$ atom and the total energy of free gold cluster $\mathrm{Au}_{n}$. using the expression, $E_{\mathrm{b}}=E_{\mathrm{T}}[\mathrm{Au}]-E_{\mathrm{T}}\left[\mathrm{Au}_{n}\right] / n$ where $\mathrm{M}$ is replaced by $\mathrm{Au}_{n}$. The binding energy of a bare gold cluster to the $\beta$-P monolayer (per supercell) is calculated as $E_{\mathrm{b}}=E_{\mathrm{T}}\left[\mathrm{Au}_{n}\right]+E_{\mathrm{T}}[\beta-\mathrm{P}]-E_{\mathrm{T}}\left[\mathrm{Au}_{n}+\beta-\mathrm{P}\right]$. As for the binding energy of a molecule to a gold cluster already adsorbed to the $\beta$-P supercell, it is calculated as $E_{\mathrm{b}}=E_{\mathrm{T}}\left[\beta-\mathrm{P}+\mathrm{Au}_{n}\right]+E_{\mathrm{T}}[M]-E_{\mathrm{T}}\left[\beta-\mathrm{P}+\mathrm{M}+\mathrm{Au}_{n}\right]$, where $E_{\mathrm{T}}\left[\beta-\mathrm{P}+\mathrm{M}+\mathrm{Au}_{n}\right]$ is the total energy of the molecule adsorbed to a gold cluster, which is already adsorbed to a $\beta$-P supercell.

The effect of solvent was deduced from calculation performed using the VASPsol ${ }^{60,61}$ code by taking $\mathrm{H}_{2} \mathrm{O}$ as solvent with the relative permittivity of $\varepsilon=80$. The band gaps are corrected over the PBE results by using the Heyd-ScuseriaErnzerhof (HSE) hybrid functional. ${ }^{62}$ The screening length of HSE is $0.2 \AA$, and the mixing rate of the HF exchange potential is 0.25 .

\section{Results and discussion}

\subsection{Binding of free AA and NB molecules to bare $\beta-P$ monolayer}

Three free AA molecules considered in this paper, i.e. glutamine $\left(\mathrm{C}_{5} \mathrm{H}_{10} \mathrm{~N}_{2} \mathrm{O}_{3}\right)$, histidine $\left(\mathrm{C}_{6} \mathrm{H}_{9} \mathrm{~N}_{3} \mathrm{O}_{2}\right)$, and tyrosine $\left(\mathrm{C}_{9} \mathrm{H}_{11} \mathrm{NO}_{3}\right)$, all have wide HOMO-LUMO gap and average cohesive energy $\bar{E}_{\mathrm{c}}$ ranging between $4.7 \mathrm{eV}$ and $5.3 \mathrm{eV} .{ }^{31}$ They are non-planar molecules. Glutamine is the most abundant free and versatile amino acid in human blood. It has fundamental importance to intermediary metabolism, interorgan nitrogen exchange via ammonia $\left(\mathrm{NH}_{3}\right)$ transport between tissues, and $\mathrm{pH}$ homeostasis. ${ }^{63}$ Histidine is a semi-essential amino acid needed in humans for growth and tissue repair. It is reported that histidine can increase the sensitivity of cancer cells to methotrexate which is an anticancer drug and lowers toxicity. ${ }^{64}$ Tyrosine is an aminocid used by cells to synthesize proteins.


Fig. 1 (a) Top and side view of the optimized atomic structure of $\beta-P$ monolayer presented for the $(5 \times 5)$ supercell. The primitive unit cell is shaded. (b) Energy band structures and indirect band gaps are calculated by PBE and HSE along symmetry directions. Total and orbital projected densities of states (DOS) are also shown.

It can transform into an enzyme of tyrosine kinase which suppresses brain tumor cell growth. ${ }^{65,66}$

Adenine $\left(\mathrm{C}_{5} \mathrm{H}_{5} \mathrm{~N}_{5}\right)$, thymine $\left(\mathrm{C}_{5} \mathrm{H}_{6} \mathrm{~N}_{2} \mathrm{O}_{2}\right)$ and guanine $\left(\mathrm{C}_{5} \mathrm{H}_{5} \mathrm{~N}_{5} \mathrm{O}\right)$ are the nucleic acids of DNA or NB used to store, transfer and express genetic information. The main body of these molecules is planar and they have $\sim 3.8 \mathrm{eV}$ HOMOLUMO gap.

Bare $\beta-\mathrm{P}^{52,53}$ monolayer has a buckled honeycomb structure (see Fig. 1(a)), where alternating $\mathrm{P}$ atoms of hexagons are buckled by $\Delta=1.23 \AA$. $\beta$-P is a semiconductor with an indirect band gap of $1.88 \mathrm{eV}$ (calculated by PBE, ${ }^{56} 2.77 \mathrm{eV}$ calculated by $\mathrm{HSE}^{62}$ in this study, see Fig. 1(b)). Its high intrinsic carrier mobility at room temperature enables nanoelectronic applications $;{ }^{67}$ its magnetic properties attained through the substitution of foreign atoms makes spintronic applications possible. ${ }^{68}$ Band gap modulations and $\mathrm{p}-\mathrm{n}$ type semiconductor engineering of $\alpha-\mathrm{P}$ and $\beta-\mathrm{P}$ monolayers via charge transfer from specific molecules have been revealed. ${ }^{69}$ Fully hydrogenated and halogenated blue phosphorenes are 2D Dirac materials which are attractive for fabricating high performance nanoelectric devices. $^{70}$ Notably, it exhibits adsorption capability to foreign atoms better than graphene, $\mathrm{MoS}_{2}, \mathrm{BN}$ and SiC. ${ }^{71}$

After this brief background on AA and NB molecules, and characterization of the $\beta$-P monolayer through the methods of calculation described in the forgoing section and used for the rest of this study, we next examine the interactions (including highest binding energy site, binding configuration and binding energy) of these molecules with bare $\beta$-P monolayer. In order to mimic the binding of a single, isolated molecule on the substrate $\beta$-P monolayer and hence to avoid intermolecular interactions, we considered one molecule adsorbed to each $(5 \times 5)$ supercell of the substrate. Possible sites of binding are the hollow, bridge and top sites of the honeycomb structure. When placed about these sites at specific orientations and heights, molecules are expected to be reoriented and translated upon structure optimization by way of the minimization of the total energy of the molecule + substrate system and atomic forces. However, the weak vdW interaction between the molecule and substrate may not generate strong enough restoring forces to reorient the molecule by series displacements and rotations in the course of the structure optimization process based on the conjugate gradient (CG) method to find the 
highest binding energy configuration; the molecule can be trapped in a local minimum. Under these circumstances the equilibrium (strongest) binding configuration of the AA and NB molecules on the $\beta-\mathrm{P}$ monolayer are determined by further minimizing the total energies for their diverse locations and orientations about positions unveiled through CG optimizations. Top and side views of molecules adsorbed on the $\beta$-P monolayer, their binding energies and minimum heights after structure optimizations are presented in Fig. 2(a). Notably, the binding energies of $\mathrm{AA}$ and $\mathrm{NB}$ molecules ranging between $460 \mathrm{meV}$ and $620 \mathrm{meV}$, are small and suggest physisorption via van der Waals (vdW) interaction. Guanine has the strongest binding with $E_{\mathrm{b}}=0.62 \mathrm{eV}$. As a result of weak vdW interaction, these molecules prefer to orient themselves parallel to the substrate so that their binding to the substrate through polarization forces is maximized. With this weak interaction, no significant effects of physisorption are expected on the mechanical and electronic structure of the molecule + substrate system. Compared to the binding energies calculated for bare black phosphorene monolayer, ${ }^{31}$ the binding energies here are generally 100-200 meV smaller.

To further understand the character of the interaction between an NB molecule and the $\beta$-P surface we let the guanine molecule rotate both clockwise and counter clockwise in a small angle about its equilibrium position, while it retains its configuration parallel to the substrate. In Fig. 2(b) we present the variation of the minimum total energy $E_{\mathrm{T}}(\theta, z)$ with the rotation angle changing in the interval of $-8^{\circ}$ (or $\left.+0.14 \mathrm{rad}\right) \geq$ $\alpha \leq 8^{\circ}(-0.14 \mathrm{rad})$ at the optimized height $z$. We see that the energy variation is rather flat and increases only $\sim 6 \mathrm{meV}$. Since guanine is rotating on the surface of $\beta$-P consisting of a buckled plane of $\mathrm{P}$ atoms at different heights, the minima of $E_{\mathrm{T}}(\theta, z)$ can deviate slightly from the smooth parabola representing a simple harmonic potential. Nevertheless, we fit this energy variation to a parabola around the global minimum to examine the rotational dynamics within the harmonic approximation. Apparently, that small energy variation is unable to generate strong enough restoring forces to reorient the molecule to its

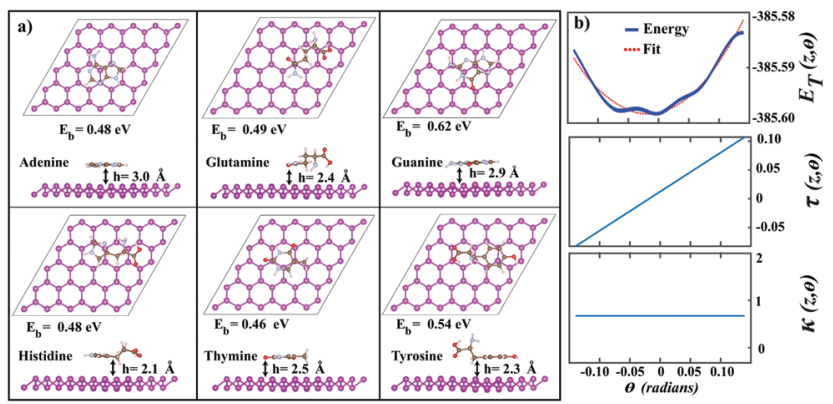

Fig. 2 (a) Top and side views of the equilibrium binding structures of three NB molecules (in the first row) and three AA molecules (in the second row). Highest binding energies $E_{\mathrm{b}}$, corresponding to minimum total energies taken as the equilibrium configurations and minimum heights $h$ are also given. (b) Variation of total energy $E_{T}(\theta, z)(\mathrm{meV})$ and its fit within the harmonic approximation, torque $\tau\left(\mathrm{eV} \mathrm{rad}^{-1}\right)$ and angular force constant $\kappa\left(\mathrm{eV} \mathrm{rad}^{-2}\right)$ calculated for guanine about their equilibrium by $\pm 8^{\circ}$. global minimum in the structure optimization process. By this applied rotation the average restoring torque, $\tau_{\mathrm{r}}=-\partial E_{\mathrm{T}} / \partial \theta$ $\left(\mathrm{eV} \mathrm{rad}^{-1}\right)$ and angular force constant, $\kappa=\tau_{\mathrm{r}} / \alpha \simeq 0.68$ $\left(\mathrm{eV} \mathrm{rad}^{-2}\right)$. Clearly, the induced restoring torque and corresponding angular force constant are too small and give rise to small libration frequency, $f=1 / 2 \pi \sqrt{\kappa / I} \simeq 0.46 \mathrm{THz}$ or angular frequency $\omega=\omega=\sqrt{\kappa / I} \simeq 3.04 \times 10^{12} \mathrm{rad} \mathrm{s}^{-1}$. This weak interaction between guanine and $\beta$-P becomes also decisive on the self-assembly behavior of the system.

\subsection{Self-assembly behavior and solvent effect}

Self-assembly of specific molecules on a substrate can be described as the phenomenon that these molecules attached to the surface assemble themselves to form a regular and stable layer or structure without any external intervention. ${ }^{72}$ The sources of self-assembly are usually molecule-substrate and molecule-molecule interactions in terms of strong chemical bonds or weak and attractive vdW forces, hydrogen bonding, electrostatic forces etc. ${ }^{73}$ Since the last few decades, the selfassembly process has gained importance in biological processes and biomaterials. ${ }^{74-78}$ The study of the self-assembly of large organic molecules on $\alpha$-P monolayers has suggested the epitaxial growth of organic thin films. ${ }^{79}$

Here, we investigate the self-assembly behavior of an NB molecule as a prototype, that is guanine found to be the most stable molecule on a $\beta$-P substrate. First, we considered the isolated molecule to reveal whether vdW interaction by itself is capable of fixing it on the substrate at a well-defined configuration. To this end we started with the configuration optimized in a $(5 \times 5)$ supercell with minute molecule-molecule coupling as shown in Fig. 2(a) and then rotated the molecule either in a horizontal or perpendicular plane above the substrate surface about the equilibrium position with large angles. This mimics a random approach of the molecule to the substrate. Subsequently, we carried out full structure optimization for molecule + substrate by minimizing the total energy and atomic forces. In the absence of the coupling with neighboring molecules, guanine did not attain a well-defined and unique configuration easily and hence did not exhibit a self-assembly behavior as quantified in the foregoing section. This is due to the very flat and shallow minimum around the equilibrium position depicted in Fig. 2(b) obtained after an extensive search in the foregoing subsection.

To further examine the self-assembly behavior, we examine the robustness of the equilibrium configuration by simulated annealing. Starting from the equilibrium configuration we raised the temperature of isolated guanine physisorbed to $\beta-\mathrm{P}$ suddenly to $500 \mathrm{~K}$ and kept the system at this temperature for 1 ps using ab initio molecular dynamics. This way, the system has been excited and departed from the minimum energy configuration. Later we reduced the temperature quickly to $0 \mathrm{~K}$ within $0.3 \mathrm{ps}$. At the end of this process guanine did not find the original equilibrium position. Clearly, the simulated annealing and other tests performed above indicate that the equilibrium positions of $\mathrm{AA}$ and NB molecules physisorbed to 
$\beta$-P individually lie in a rather shallow minimum (minima) and they cannot acquire a unique configuration and thus they do not show strict self-assembly behavior.

To reveal the effect of molecule-molecule interaction, we optimized four guanines placed first randomly in a $(6 \times 6)$ supercell of $\beta$-P substrate as shown in Fig. 3(a). Upon optimization, guanine kept its parallel orientation to the surface, but displaced/rotated and eventually attained a well-defined orientation, whereby the binding energy per molecule is increased by $\sim 200 \mathrm{meV}$ due to intermolecular coupling. This coupling energy shall increase with increasing coverage due to molecule-molecule interaction. Also, it is deduced that the configuration or order of physisorbed guanines will depend on their coverage, as well as on the initial conditions.

The effect of substrate-molecule interactions has been examined further by removing the substrate holding four guanine molecules initially in a cell of $(6 \times 6)$, and then optimizing the structure. In the absence of substrate these molecules underwent significant displacements and reorientations to minimize their total energy as shown in Fig. 3(b). At the end, the magnitude of the average total energy relative to free molecules is increased by $\sim 0.5 \mathrm{eV}$. Since the underlying substrate is absent, the binding is constructed due to merely molecule-molecule coupling; the average binding energy thereof is comparable to the binding of individual guanine physisorbed to $\beta$-P. These results suggest that guanine and other molecules here may display a kind of self-assembly at high coverage on the surface of $\beta$-P, even though it lacks regularity. Regular self-assembly with most of the anchored molecules displaying the same configuration/orientation requires stronger molecule-substrate interaction, which can be provided by the adsorption of adatoms or cluster like gold as we discuss in the forthcoming sections.

In practice, the organic molecules are treated here to coexist with the solvent on the surfaces. To investigate the solvent effect on a $\beta$ P-guanine system, we use the continuum solvation (or implicit solvation) model, in which the vacuum space is filled with the dielectric continuum corresponding to the solvent. ${ }^{60}$ Here we consider only water as a solvent with a suitable dielectric constant. In solvation calculation, the total energy decrease $(800 \mathrm{meV})$ and the morphology of the substrate-molecule system do not change visibly. Here we address

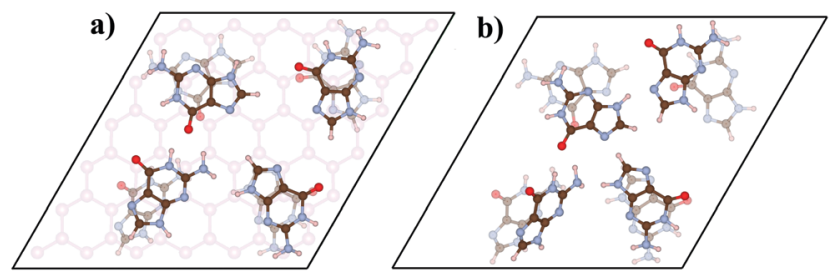

Fig. 3 Self-assembly behavior of guanine molecules. (a) Initial (pale) and final optimized configurations (dark) of four guanine molecules placed on a $\beta$-P substrate in its $(6 \times 6)$ supercell. Structures repeat periodically within the supercell geometry. (b) Initial positions (pale), which are the same as (a), and final optimized configurations of four guanine molecules without a substrate. the question of how the binding energy of guanine changes in the solvent. Normally, the binding energy is required to take the physisorbed molecule from its equilibrium position to infinity. To present a reasonable estimate for the binding energy of guanine in water, we compared the optimized total energy of guanine, which is physisorbed to $\beta$-P in equilibrium in the solvent and of guanine, which is fixed at a large distance (9.0 $)$ above the surface of a $\beta$-P monolayer again in the solvent. The difference in the total energies of these two systems, which is taken as a good estimate for the binding energy, was found to be $\sim 340 \mathrm{meV}$. By assuming that the binding energy of guanine in water is negligible when it is increased to a height of $9.0 \AA$, we deduce that its binding energy to blue phosphorene in water decreases and becomes $E_{\mathrm{b}} \sim 340 \mathrm{meV}$. Within the continuum solvation model, we expect the same effect of solvent in the binding of other molecules.

\subsection{Adsorption of gold atoms and gold clusters on bare $\boldsymbol{\beta}-\mathbf{P}$}

The adsorption of a single gold atom is critical for the functionalization of phosphorene surfaces. Our motivation in considering the adsorption of gold atoms and gold clusters to $\beta-\mathrm{P}$ is whether we can attain stronger binding of AA and NB molecules and realize their fixation. However, before we present a detailed analysis of the coverage of gold clusters, $\mathrm{Au}_{n}(n=1-5$, including single $\mathrm{Au}$ atom) and their interactions with AA and NB molecules on the $\beta$-P surface, we first examine the coverage dependent properties of $\beta$-P with Au atoms. Here, we considered the gold atom coverages, $\theta=1,1 / 2,1 / 3,1 / 4$ and $1 / 5$ using the supercell geometry, corresponding to $(1 \times 1),(2 \times 2),(3 \times 3),(4 \times 4)$ and $(5 \times 5)$, respectively. This way, we were able to treat the adatom-adatom coupling in a wide range from strong to very weak. Upon extensive geometry/structure optimization analysis we found that while the top site of host $\mathrm{P}$ atom (namely each $\mathrm{P}$ atom in the primitive unit cell of $\beta-\mathrm{P}$, which is increased upon buckling) is the equilibrium location of $\mathrm{Au}$ atoms for $\theta=1$, the hollow site (i.e. above the centers of $\mathrm{P}$ hexagons) becomes the equilibrium site for $\theta=1 / 2-1 / 5$.

The energy band structures of $\beta$-P for different coverages $(\theta=1-1 / 5)$ of $\mathrm{Au}$ atoms are presented in Fig. 4. For full coverage, i.e. $\theta=1$ the semiconducting surface changes to a nonmagnetic metal with a high density of states at $E_{\mathrm{F}}$. The binding energy of a Au atom, $E_{\mathrm{b}}=2.96 \mathrm{eV}$, is rather high due to $\mathrm{Au}-\mathrm{Au}$ coupling of the nearest adsorbed atoms. For the same reason, one surface of $\beta$-P, which is fully covered by Au adatoms is not dynamically stable as revealed from severe instability in specific phonon modes. This situation indicates that $\mathrm{Au}$ atoms prefer to form clusters on the surface due to $\mathrm{Au}-\mathrm{Au}$ interaction being much stronger than that of Au- $\beta-\mathrm{P}$ (only $\sim 0.80 \mathrm{eV}$ ). The high density of electronic states at the Fermi level is another manifestation of structural instability. For the coverage $\theta=1 / 2$ corresponding to one $\mathrm{Au}$ atom adsorbed to a specific hollow site of each $(2 \times 2)$ supercell, the binding energy becomes $E_{\mathrm{b}}=1.82 \mathrm{eV}$ because of reduced $\mathrm{Au}-\mathrm{Au}$ coupling and the $\mathrm{Au}+\beta-\mathrm{P}$ system acquires a permanent magnetic moment of $1.0 \mu_{\mathrm{B}}$ whereby the high density of states split with a band gap. According to the spinresolved band structure, the system is a semiconductor for both 

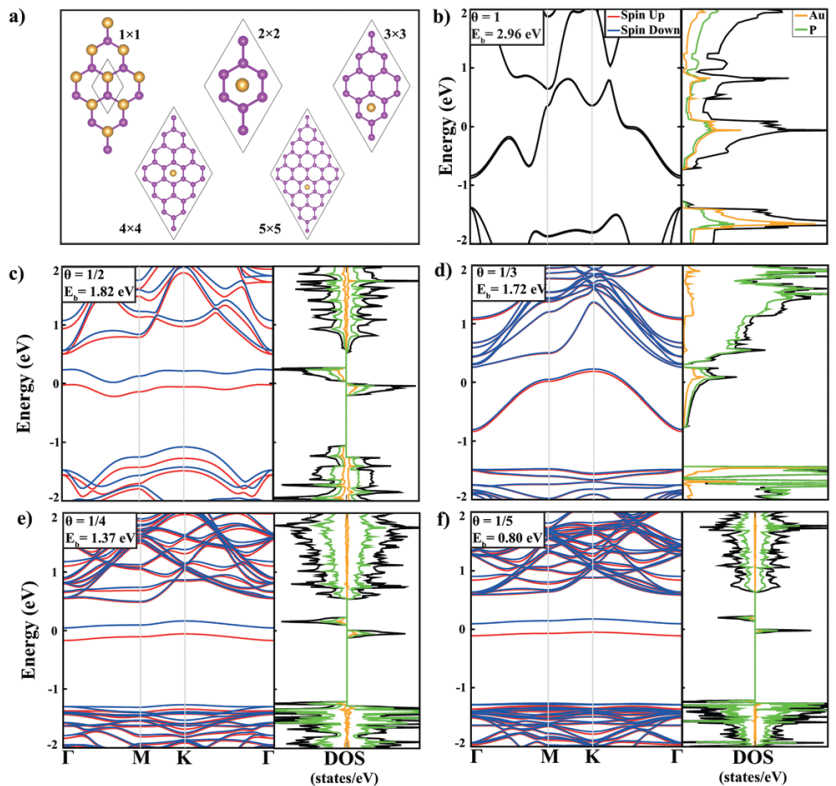

Fig. 4 Spin-polarized, electronic energy band structures of $\mathrm{Au}$ atoms adsorbed to $\beta-P$ in various coverages, $\theta=1,1 / 2,1 / 3,1 / 4$ and $1 / 5$, and the corresponding orbital projected and spin-projected densities of states. The zero of energy is taken at the Fermi level. The supercells corresponding to these specific coverage values with top and hollow adsorption sites are shown by insets.

majority and minority spin electrons. However, there is no gap between the majority electrons in the valence band and minority electrons in the conduction band. Accordingly, this system is specified as a spin gapless semiconductor. ${ }^{80}$ In contrast, the coverage $\theta=1 / 3$ has spin-resolved bands which are hardly split and thus has minute magnetic moment. The binding energy of $\mathrm{Au}$ adatoms continues to reduce because of increasing adatom-adatom distance. The situation for $\theta=1 / 4$ is very similar to $\theta=1 / 2$; its spin-resolved bands attribute a spin gapless semiconducting state to $\beta$-P systems; the supercell attained a permanent magnetic moment of $1.0 \mu_{\mathrm{B}}$ per supercell. The binding energy is further reduced to $E_{\mathrm{b}}=1.37 \mathrm{eV}$. Earlier results by Ding and Wang ${ }^{71}$ on Au coverage of blue phosphorene calculated only for $\theta=1 / 4$ are in good agreement with the present ones. The modifications of the $\beta$-P surface and the resulting electronic structure for coverage $\theta=1 / 5$ mimics the adsorption of a single (isolated) Au atom owing to negligible $\mathrm{Au}-\mathrm{Au}$ coupling. The same coverage value was used in the physisorption of AA and NB molecules on bare $\beta$-P surface. For $\theta=1 / 5, \mathrm{Au}+\beta-\mathrm{P}$ is a magnetic semiconductor for both spin directions, but a very small bad gap opens between majority electrons of the valence band and minority electrons of the conduction band. Neglecting this very small band gap, it is actually a spin gapless semiconductor. The magnetic moment per supercell is again $1.0 \mu_{\mathrm{B}}$ and the binding energy $E_{\mathrm{b}}=$ $0.80 \mathrm{eV}$. Apparently, the surface of $\beta-\mathrm{P}$ is dramatically modified by single Au coverage. Depending on the coverage or decoration, bare $\beta-P$, which is a semiconductor, is functionalized by acquiring different electronic and magnetic states.
We next investigate the adsorption of gold clusters, $\mathrm{Au}_{n}$ $(n=1-5)$, including a single $\mathrm{Au}$ atom corresponding to $(n=1)$ on the $\beta$-P surface with $\theta=1 / 5$ (namely, the same type of gold cluster is adsorbed in each $(5 \times 5)$ supercell $)$. Binding configuration and binding energy is determined by the structure optimization of the $\mathrm{Au}_{n}+\beta-\mathrm{P}$ system. The calculated binding energies and top and side views of the binding configurations of absorbed gold clusters are shown in Fig. 5. Owing to multiple interactions of $\mathrm{Au}$ atoms in the cluster with the substrate surface the binding energies increase with an increasing number of $\mathrm{Au}$ atoms, $n$.

Similar to single $\mathrm{Au}$ adatoms, $\beta-\mathrm{P}$ attains diverse electronic properties upon the coverage of various gold clusters. The binding energy, which is only $0.80 \mathrm{eV}$ for $n=1$, gradually increases due to interaction of multiple cluster atoms with the substrate and becomes $E_{\mathrm{b}}=4.14 \mathrm{eV}$ for $n=5$. Depending on the type of cluster or the value of $n$, the $\mathrm{Au}_{n}+\beta$-P system can attain magnetic moments $1.0 \mu_{\mathrm{B}}$ or $0.33 \mu_{\mathrm{B}}$ or can be nonmagnetic.

\subsection{Fixation of AA and NB molecules to adsorbed gold clusters}

Now we examine the binding of AA and NB molecules attached to these gold clusters on blue phosphorene. Determination of the equilibrium binding energy and corresponding binding geometry of these molecules is really elusive and required an extensive structure optimization process starting from diverse initial configurations. In Fig. 6 we show perspective views of AA and NB molecules adsorbed to various gold clusters on the $\beta-\mathrm{P}$ surface. Generally, AA and NB molecules prefer to be adsorbed to $\mathrm{Au}_{n}$ clusters through $\mathrm{N}-\mathrm{Au}$ or $\mathrm{O}-\mathrm{Au}$ bonding and also through both in certain circumstances. This is a consequence of the optimization of the binding configuration using a CG method, where the geometry of the outermost $\mathrm{Au}$ atoms and the charge distribution thereof play a decisive role. In the course of the optimization of the adsorption process, hydrogen atoms of carboxyl or amino group do not leave molecules even at temperatures as high as $500 \mathrm{~K}$ to form a strong chemical bond with different atoms. Since the binding configuration and the corresponding energies are calculated using $(5 \times 5)$ supercells, intermolecular interaction are minimized to mimic the adsorption of individual/isolated molecules to the clusters. The binding energies of these molecules are presented in Table 1. In comparison with the binding to bare $\beta$-P surface, the most prominent difference is that the binding of AA and NB molecules to gold clusters become 2-3 times stronger. Owing to the relatively strong chemical interaction between individual atoms

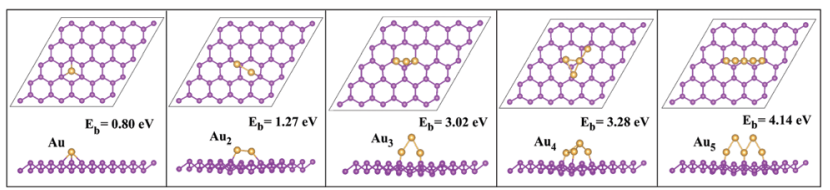

Fig. 5 Top and side views of the adsorption geometry of gold clusters $\mathrm{Au}_{n}$ $(n=1-5)$ on a $\beta$-P substrate in $\theta=1 / 5$ coverage achieved by $(5 \times 5)$ supercells of $\beta-P$. The binding energy of each cluster, $E_{\mathrm{b}}$ is also given. 

Adenine
Glutamine
Guanine
Histidine
Thymine
Tyrosine

-

$\rightarrow-0$

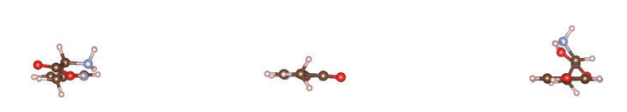

Bare
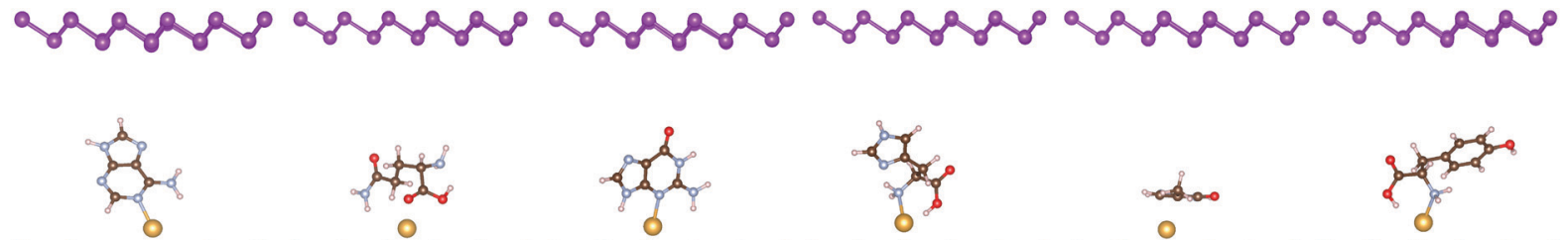

$\mathrm{Au}_{1}$

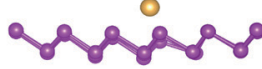

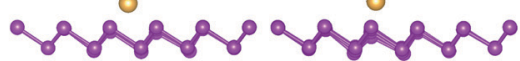

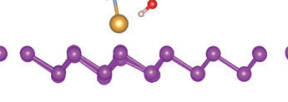

0
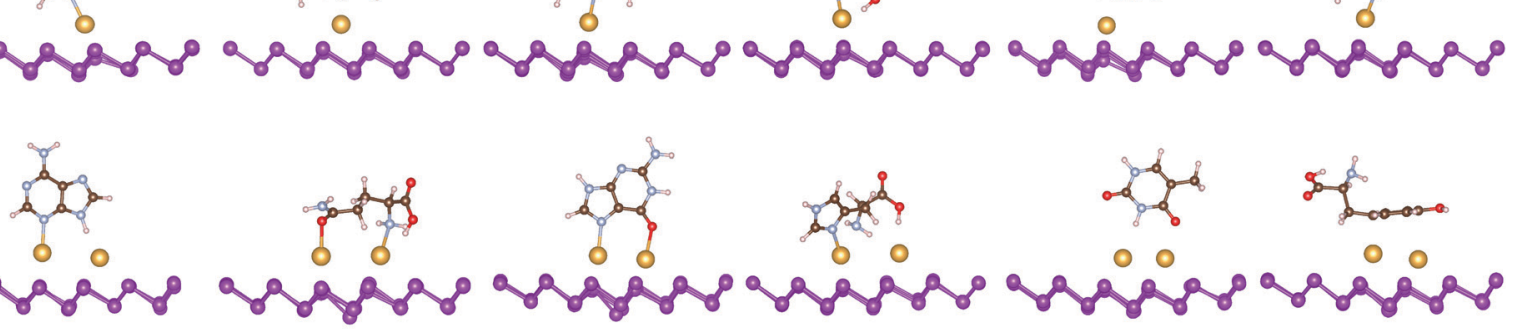

$\mathrm{Au}_{2}$


- 0
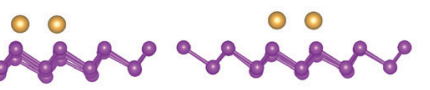

00
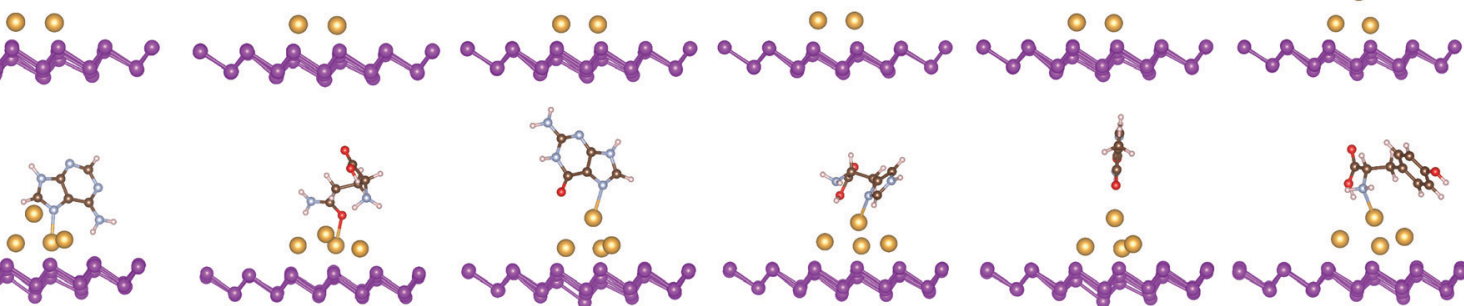

$\mathrm{Au}_{4}$


$\mathrm{Au}_{5}$

000

- 00

- 00

- 00

Fig. 6 Perspective views of optimized structures of AA and NB molecules adsorbed to bare $\beta-P$ (first row) and to the gold clusters $\left(A u_{n}\right.$ with $n=1-5$ ), which were already adsorbed to a $\beta$-P monolayer. The coverage $\theta=1 / 5$ corresponds to the same type of cluster being adsorbed to each ( $5 \times 5)$ supercell. Each equilibrium configuration presented here is determined by optimizing a number of possible adsorption geometries to find the lowest energy configuration.

Table 1 Binding energy $E_{\mathrm{b}}$ (in eV) of AA and NB molecules with $\mathrm{Au}_{n}$ ( $n=$ $1-5)$ clusters on the $\beta$-P surface

\begin{tabular}{lcccccc}
\hline A.A & $\mathrm{Bare}$ & $\mathrm{Au}_{1}$ & $\mathrm{Au}_{2}$ & $\mathrm{Au}_{3}$ & $\mathrm{Au}_{4}$ & $\mathrm{Au}_{5}$ \\
\hline Adenine & 0.48 & 1.63 & 1.65 & 1.18 & 1.81 & 1.11 \\
Glutamine & 0.50 & 0.66 & 1.50 & 0.67 & 1.29 & 1.01 \\
Guanine & 0.62 & 1.69 & 1.51 & 1.17 & 1.19 & 1.16 \\
Histidine & 0.48 & 1.41 & 1.72 & 1.18 & 0.90 & 1.63 \\
Thymine & 0.46 & 1.07 & 0.64 & 0.71 & 0.79 & 0.73 \\
Tyrosine & 0.54 & 1.24 & 0.85 & 0.81 & 1.20 & 1.39
\end{tabular}

of molecules with host $\mathrm{P}$ atoms, the molecules attained binding geometries, which are different from their physisorption to a bare $\beta$-P surface. While most of the molecules were physisorbed parallel to bare $\beta-\mathrm{P}$, molecules adsorbed to gold clusters attained equilibrium configuration with an angle to the substrate surface. Diverse configurations of outermost atoms of gold clusters interacting with AA and NB molecules can give rise to different binding energies. For example, while $\mathrm{Au}_{2}$, as well as $\mathrm{Au}_{5}$ clusters with their two reactive, outermost $\mathrm{Au}$ atoms give rise to relatively stronger bindings for most of the molecules, $\mathrm{Au}_{3}$ and in certain cases $\mathrm{Au}_{4}$ clusters with a single reactive, outermost $\mathrm{Au}$ atom may lead to relatively smaller binding energies. Nonetheless, the binding energy between AA or NB molecules and Au $n$ clusters depends on the detailed configurations of interacting atoms, which may even undergo changes relative to their configurations before adsorption.

This analysis suggests that the fixation of AA and NB molecules can be achieved by gold nanoclusters, which are 
already adsorbed to $\beta-\mathrm{P}$. The chemical bonds between a molecule and $\mathrm{Au}$ atoms of the cluster is strong enough to orient the molecule to a well-defined configuration. Via decoration of the $\beta$-P surface with a specific gold cluster, AA and NB molecules can easily be self-assembled on substrates. Through this selfassembly one can form larger and complex molecules. Also the surface of the substrate can gain desired properties through the self-assembled coating by AA or NB molecules. Finally, based on spin polarized DFT calculations we present an example for the modification of an electronic structure induced by guanine. Starting from the bare $\beta-\mathrm{P}$, which is an indirect band gap semiconductor, the physisorption of guanine does not cause any significant change in the electronic structure; the electronic structure of $\beta-\mathrm{P}+$ guanine becomes as if the energy levels of free guanine are superimposed to the $2 \mathrm{D}$ band structure of the bare $\beta$-P monolayer without any mixing or hybridization, except for a small energy shift. On the other hand, when covered by Au adatoms at $\theta=1 / 5$, the nonmagnetic, semiconducting bare $\beta$-P changes to a spin gapless semiconductor with a net magnetic moment of $1.0 \mu_{\mathrm{B}}$ per cell. If this $\mathrm{Au}$ atom absorbed to a $\beta-\mathrm{P}$ monolayer at $\theta=1 / 5$ is capped (or saturated) by guanine, the $\beta-\mathrm{P}$ monolayer $+\mathrm{Au}+$ guanine system becomes a nonmagnetic metal. These series of changes undergone by the $\beta$-P monolayer are important and promise new functionalities of $2 \mathrm{D}$ monolayers gained by the coating of organic molecules.

\section{Conclusions}

Amino acids, like glutamine, histidine and tyrosine, and nucleic acid bases, like adenine, guanine and thymine, are critical organic molecules and their interface with diverse substrate surfaces has been of interest in biomedicine. In this work, we investigated the interactions between these individual molecules and biofriendly, bare, blue phosphorene as well as blue phosphorene covered with gold clusters using state-of-the art, firstprinciples density functional theory. Here is a brief summary of our important findings followed by physical explanations:

(i) The weak van der Waals interaction between each individual molecule and monolayer is not sufficiently strong; molecules can be neither fixed to the substrate nor selfassembled on the surface. Their configuration parallel to the surface of the substrate in a shallow minimum cannot generate strong enough restoring force or torque to maintain the equilibrium configuration against the thermal excitations. Under these circumstances, their libration frequencies are rather low. On the other hand, molecule-molecule coupling due to reduced intermolecular distance at high coverage is significant. Under such weak binding, the electronic structure of the substrate, as well as the energy level structure of the molecule are not affected, except for a small relative shift. Our study using guanine as a prototype molecule suggests that the binding of these molecules to phosphorenene is further decreased in a solvent like water.

(ii) Gold atoms and gold clusters adsorbed on $\beta$-P monolayer are considered to provide active sites for fixation of molecules.
A gold adatom by itself, modifies the electronic and magnetic properties of blue phosphorene depending on the coverage and decoration. For example, depending on the gold adatom coverage, blue phosphorene monolayer can acquire nonmagnetic metallic or spin gapless semiconducting states with an integer permanent magnetic moment. However, due to strong adatom-adatom coupling, high coverage of gold adatoms is unstable, but favors clustering. Gold nanoclusters also form strong chemical bonds with a blue phosphorene monolayer and modify the electronic properties depending on the size of the clusters. In addition, individual amino acid or nucleic base molecules can be readily adsorbed to gold clusters via strong chemical bonds and be reoriented in a well-defined configuration. The calculated binding energies are high to fix the adsorbed molecule on the surface and to attain selfassembly character. Accordingly, not only is fixation of individual molecules on the substrate achieved, but also the durable coating of the substrate surface by these molecules is made possible. This way, the bare monolayer surface can now obtain new functionalities.

\section{Conflicts of interest}

There are no conflicts to declare.

\section{Acknowledgements}

Computing resources used in this work were provided by the TUBITAK ULAKBIM, High Performance and Grid Computing Center (Tr-Grid e-Infrastructure). S. C. acknowledges financial support from the Academy of Sciences of Turkey TÜBA.

\section{References}

1 J. Rush, A. Moritz, K. A. Lee, A. Guo, V. L. Goss, E. J. Spek, H. Zhang, X.-M. Zha, R. D. Polakiewicz and M. J. Comb, Nat. Biotechnol., 2005, 23, 94-101.

2 D. R. Wise and C. B. Thompson, Trends Biochem. Sci., 2010, 35, 427-433.

3 O. Cantoni, P. Sestili, A. Guidarelli, P. U. Giacomoni and F. Cattabeni, Mol. Pharmacol., 1992, 41, 969-974.

4 R. Misra, S. Acharya and S. K. Sahoo, Drug Discovery Today, 2010, 15, 842-850.

5 A. P. Singh, S. Senapati, M. P. Ponnusamy, M. Jain, S. M. Lele, J. S. Davis, S. Remmenga and S. K. Batra, Lancet Oncol., 2008, 9, 1076-1085.

6 A. Bolhassani, S. Safaiyan and S. Rafati, Mol. Cancer, 2011, 10, 1-20.

7 H. Ren, D. D. Kulkarni, R. Kodiyath, W. Xu, I. Choi and V. V. Tsukruk, ACS Appl. Mater. Interfaces, 2014, 6, 2459-2470.

8 D. Chen, L. Li and L. Guo, Nanotechnology, 2011, 22, 325601.

9 P. Rubio-Pereda and G. H. Cocoletzi, Appl. Surf. Sci., 2018, 427, 1227-1234.

10 D. Cortes-Arriagada, J. Phys. Chem. C, 2018, 122, 4870-4880. 
11 H.-p. Zhang, X.-y. Lin, X. Lu, Z. Wang, L. Fang and Y. Tang, Mater. Chem. Front., 2017, 1, 1156-1164.

12 F. Ersan, O. Uzengi Akturk, E. Akturk and S. Ciraci, J. Phys. Chem. C, 2018, 122, 14598-14605.

13 D. Li, W. Zhang, X. Yu, Z. Wang, Z. Su and G. Wei, Nanoscale, 2016, 8, 19491-19509.

14 P. Zhang, H. Wang, X. Zhang, W. Xu, Y. Li, Q. Li, G. Wei and Z. Su, Biomater. Sci., 2015, 3, 852-860.

15 Y. Wang, Z. Li, D. Hu, C.-T. Lin, J. Li and Y. Lin, J. Am. Chem. Soc., 2010, 132, 9274-9276.

16 K. Kalantar-zadeh and J. Z. Ou, ACS Sens., 2016, 1, 5-16.

17 K. Huang, Z. Li, J. Lin, G. Han and P. Huang, Chem. Soc. Rev., 2018, 47, 5109-5124.

18 H. Zhao, R. Ding, X. Zhao, Y. Li, L. Qu, H. Pei, L. Yildirimer, Z. Wu and W. Zhang, Drug Discovery Today, 2017, 22, 1302-1317.

19 P. Suvarnaphaet and S. Pechprasarn, Sensors, 2017, 17, 2161.

20 H. Berney, J. West, E. Haefele, J. Alderman, W. Lane and J. Collins, Sens. Actuators, B, 2000, 68, 100-108.

21 N. Sanvicens, C. Pastells, N. Pascual and M.-P. Marco, TrAC, Trends Anal. Chem., 2009, 28, 1243-1252.

22 A. B. Farimani, K. Min and N. R. Aluru, ACS Nano, 2014, 8, 7914-7922.

23 L. Xiao, L. Xu, C. Gao, Y. Zhang, Q. Yao and G.-J. Zhang, Sensors, 2016, 16, 1561.

24 S. Feng, C. Cong, N. Peimyoo, Y. Chen, J. Shang, C. Zou, B. Cao, L. Wu, J. Zhang and M. Eginligil, et al., Nano Res., 2018, 11, 1744-1754.

25 J.-H. Lee, Y.-K. Choi, H.-J. Kim, R. H. Scheicher and J.-H. Cho, J. Phys. Chem. C, 2013, 117, 13435-13441.

26 K. Janani and D. J. Thiruvadigal, Appl. Surf. Sci., 2018, 449, 829-837.

27 Z. Li, Y. Chen, X. Li, T. Kamins, K. Nauka and R. S. Williams, Nano Lett., 2004, 4, 245-247.

28 F. R. Rahsepar and K. T. Leung, J. Phys. Chem. C, 2018, 122, 16113-16121.

29 O. Ourdjini, R. Pawlak, M. Abel, S. Clair, L. Chen, N. Bergeon, M. Sassi, V. Oison, J.-M. Debierre and R. Coratger, et al., Phys. Rev. B: Condens. Matter Mater. Phys., 2011, 84, 125421.

30 M. B. Radoičić, I. A. Janković, V. N. Despotović, D. V. Šojić, T. D. Savić, Z. V. Šaponjić, B. F. Abramović and M. I. Čomor, Appl. Catal., B, 2013, 138, 122-127.

31 Y. Kadioglu, T. Gorkan, O. Üzengi Aktürk, E. Aktürk and S. Ciraci, J. Phys. Chem. C, 2019, 123, 23691-23704.

32 C. D. Medley, J. E. Smith, Z. Tang, Y. Wu, S. Bamrungsap and W. Tan, Anal. Chem., 2008, 80, 1067-1072.

33 S.-f. Liu, Y.-f. Li, J.-r. Li and L. Jiang, Biosens. Bioelectron., 2005, 21, 789-795.

34 A. N. Shipway, E. Katz and I. Willner, ChemPhysChem, 2000, 1, 18-52.

35 W. H. Lee, J. Park, S. H. Sim, S. Lim, K. S. Kim, B. H. Hong and K. Cho, J. Am. Chem. Soc., 2011, 133, 4447-4454.

36 F. Ersan, E. Aktürk and S. Ciraci, Carbon, 2019, 143, 329-334.

37 S.-Y. Wu, S. S. A. An and J. Hulme, Int. J. Nanomed., 2015, 10, 9 .
38 B. Zhang, P. Wei, Z. Zhou and T. Wei, Adv. Drug Delivery Rev., 2016, 105, 145-162.

39 X. Tan, L. Feng, J. Zhang, K. Yang, S. Zhang, Z. Liu and R. Peng, ACS Appl. Mater. Interfaces, 2013, 5, 1370-1377.

40 Y.-n. Guo, X. Lu, J. Weng and Y. Leng, J. Phys. Chem. C, 2013, 117, 5708-5717.

41 J. MacLeod and F. Rosei, Small, 2014, 10, 1038-1049.

42 J. Katoch, S. N. Kim, Z. Kuang, B. L. Farmer, R. R. Naik, S. A. Tatulian and M. Ishigami, Nano Lett., 2012, 12, 2342-2346.

43 N. Dragneva, O. Rubel and W. B. Floriano, J. Chem. Inf. Model., 2016, 56, 706-720.

44 S. Dong, M. Zeng, D. Wang, Z. Liu, Y. Zhao and H. Yang, Food Chem., 2008, 107, 1485-1493.

45 G. Liang, Z. Yang, R. Zhang, L. Li, Y. Fan, Y. Kuang, Y. Gao, T. Wang, W. W. Lu and B. Xu, Langmuir, 2009, 25, 8419-8422.

46 E. A. Wang, V. Rosen, J. S. D’Alessandro, M. Bauduy, P. Cordes, T. Harada, D. I. Israel, R. M. Hewick, K. M. Kerns and P. LaPan, Proc. Natl. Acad. Sci. U. S. A., 1990, 87, 2220-2224.

47 G. Johnston and L. Davies, J. Neurochem., 1974, 22, 101-105. 48 X. Yang, X. Zhang, Z. Liu, Y. Ma, Y. Huang and Y. Chen, J. Phys. Chem. C, 2008, 112, 17554-17558.

49 D. Depan, J. Shah and R. Misra, Mater. Sci. Eng., C, 2011, 31, 1305-1312.

50 Y. Chong, C. Ge, Z. Yang, J. A. Garate, Z. Gu, J. K. Weber, J. Liu and R. Zhou, ACS Nano, 2015, 9, 5713-5724.

51 S. Kumar and S. H. Parekh, Commun. Chem., 2020, 3, 1-11. 52 J. L. Zhang, S. Zhao, C. Han, Z. Wang, S. Zhong, S. Sun, R. Guo, X. Zhou, C. D. Gu and K. D. Yuan, et al., Nano Lett., 2016, 16, 4903-4908.

53 Z. Zhu and D. Tománek, Phys. Rev. Lett., 2014, 112, 176802.

54 G. Kresse and J. Furthmüller, Phys. Rev. B: Condens. Matter Mater. Phys., 1996, 54, 11169.

55 G. Kresse and J. Furthmüller, Comput. Mater. Sci., 1996, 6, 15-50.

56 J. P. Perdew, K. Burke and M. Ernzerhof, Phys. Rev. Lett., 1996, 77, 3865.

57 P. E. Blöchl, Phys. Rev. B: Condens. Matter Mater. Phys., 1994, 50, 17953.

58 G. Kresse and D. Joubert, Phys. Rev. B: Condens. Matter Mater. Phys., 1999, 59, 1758.

59 S. Grimme, J. Comput. Chem., 2006, 27, 1787-1799.

60 K. Mathew, R. Sundararaman, K. Letchworth-Weaver, T. Arias and R. G. Hennig, J. Chem. Phys., 2014, 140, 084106.

61 M. Fishman, H. L. Zhuang, K. Mathew, W. Dirschka and R. G. Hennig, Phys. Rev. B: Condens. Matter Mater. Phys., 2013, 87, 245402.

62 J. Heyd, G. E. Scuseria and M. Ernzerhof, J. Chem. Phys., 2003, 118, 8207-8215.

63 V. Cruzat, M. Macedo Rogero, K. Noel Keane, R. Curi and P. Newsholme, Nutrients, 2018, 10, 1564.

64 N. Kanarek, H. R. Keys, J. R. Cantor, C. A. Lewis, S. H. Chan, T. Kunchok, M. Abu-Remaileh, E. Freinkman, L. D. Schweitzer and D. M. Sabatini, Nature, 2018, 559, 632-636. 
65 E. Hatva, A. Kaipainen, P. Mentula, J. Jääskeläinen, A. Paetau, M. Haltia and K. Alitalo, Am. J. Pathol., 1995, 146, 368.

66 P. Vajkoczy, P. Knyazev, A. Kunkel, H.-H. Capelle, S. Behrndt, H. von Tengg-Kobligk, F. Kiessling, U. Eichelsbacher, M. Essig and T.-A. Read, et al., Proc. Natl. Acad. Sci. U. S. A., 2006, 103, 5799-5804.

67 J. Xiao, M. Long, X. Zhang, J. Ouyang, H. Xu and Y. Gao, Sci. Rep., 2015, 5, 9961.

68 M. Sun, W. Tang, Q. Ren, S.-k. Wang, J. Yu and Y. Du, Appl. Surf. Sci., 2015, 356, 110-114.

69 C. Chowdhury, S. Jahiruddin and A. Datta, J. Phys. Chem. Lett., 2016, 7, 1288-1297.

70 M. Sun, W. Tang, Q. Ren, S.-k. Wang, J. Yu and Y. Du, Appl. Surf. Sci., 2015, 356, 110-114.

71 Y. Ding and Y. Wang, J. Phys. Chem. C, 2015, 119, 10610-10622.
72 G. M. Whitesides and B. Grzybowski, Science, 2002, 295, 2418-2421.

73 C. J. Brinker, Y. Lu, A. Sellinger and H. Fan, Adv. Mater., 1999, 11, 579-585.

74 X. Li, X. Yang, J. Qi and N. C. Seeman, J. Am. Chem. Soc., 1996, 118, 6131-6140.

75 E. Winfree, F. Liu, L. A. Wenzler and N. C. Seeman, Nature, 1998, 394, 539-544.

76 R. Chhabra, J. Sharma, Y. Liu, S. Rinker and H. Yan, Adv. Drug Delivery Rev., 2010, 62, 617-625.

77 J. Sharma, R. Chhabra, A. Cheng, J. Brownell, Y. Liu and H. Yan, Science, 2009, 323, 112-116.

78 T. K. Mukhopadhyay and A. Datta, J. Phys. Chem. C, 2018, 122, 28918-28933.

79 T. K. Mukhopadhyay and A. Datta, J. Phys. Chem. C, 2017, 121, 10210-10223.

80 X. Wang, Phys. Rev. Lett., 2008, 100, 156404. 